\title{
28 Research Square \\ 18f-fdg Pet/mr in Focal Epilepsy: a New Step for Improving the Detection of Epileptogenic Lesions
}

\section{Anthime FLAUS}

SHFJ: Commissariat a l'energie atomique et aux energies alternatives Service Hospitalier Frederic Joliot

\section{Charles MELLERIO}

GHU Paris: Groupe Hospitalier Universitaire Paris psychiatrie \& neurosciences

\section{Sebastian RODRIGO}

SHFJ: Commissariat a l'energie atomique et aux energies alternatives Service Hospitalier Frederic Joliot

\section{Vincent BRULON}

SHFJ: Commissariat a l'energie atomique et aux energies alternatives Service Hospitalier Frederic Joliot

\section{Vincent LEBON}

SHFJ: Commissariat a l'energie atomique et aux energies alternatives Service Hospitalier Frederic Joliot

Francine CHASSOUX ( $\nabla$ f.chassoux@ghu-paris.fr )

Centre Hospitalier Sainte Anne https://orcid.org/0000-0002-1970-6578

\section{Original research}

Keywords: 18F-FDG PET, PET/MR, Drug-resistant Focal Epilepsy, Epileptogenic Zone, Focal Cortical Dysplasia, Epilepsy Surgery

Posted Date: March 3rd, 2021

DOI: https://doi.org/10.21203/rs.3.rs-254510/v1

License: (c) (i) This work is licensed under a Creative Commons Attribution 4.0 International License. Read Full License

Version of Record: A version of this preprint was published at Epilepsy Research on November 1st, 2021. See the published version at https://doi.org/10.1016/j.eplepsyres.2021.106819. 


\section{Abstract}

Purpose: Hybrid PET/MR is a promising tool in focal drug-resistant epilepsy, however the additional value for the detection of epileptogenic lesions and surgical decision-making remains to be established.

Methods: We retrospectively compared ${ }^{18} \mathrm{~F}-\mathrm{FDG}$ PET/MR images with those obtained by a previous ${ }^{18} \mathrm{~F}-\mathrm{FDG}$ PET coregistered with MRI (PET+MR) in 25 consecutive patients (16 females, 13-60 year-old) investigated for focal drugresistant epilepsy. Visual analysis was performed by two readers blinded from imaging modalities, asked to assess the technical characteristics (co-registration, quality of images), confidence in results, location of PET abnormalities and presence of a structural lesion on MRI. The clinical impact on surgical strategy and outcome was assessed independently.

Results: The location of epilepsy was temporal in 9 patients and extra-temporal in 16 others. MRI was initially considered negative in 21 of them. PET alone demonstrated metabolic abnormalities in 19 cases (76\%), and the coregistration with MRI allowed the detection of 4 additional structural lesions. PET/MR was considered better performing than PET+MR in $56 \%$ of patients. The increase in sensitivity was $13 \%$ and new structural lesions (mainly focal cortical dysplasias) were detected in 6 patients (24\%). Change of surgical decision-making was substantial for $40 \%$ of patients, consisting in avoiding invasive monitoring in 6 patients and modifying the planning in 4 others. Seizure-free outcome was obtained in 13/14 patients who underwent a cortical resection.

Conclusion: Hybrid PET/MR improves the detection of epileptogenic lesions, allowing to optimize the presurgical workup and to increase the proportion of successful surgery even in the more complex cases.

\section{Introduction}

The detection of epileptogenic lesions is crucial for identifying the best candidates for surgery in drug-resistant focal epilepsy. Among the different types of lesions causing epilepsy, focal cortical dysplasias (FCDs) represent one of the most frequent both in children and in adults [1]. Surgical resection of the dysplastic cortex allows a favorable outcome [2-3], however FCDs can be difficult to identify by imaging even though using optimal MRI protocols [4-7]. ${ }^{18}$ F-FDG PET has proved to be useful in negative MRI cases, and the additional value of the co-registration of PET and MRI has been demonstrated [8-13]. More recently, new hybrid PET/MR imaging has been developed, allowing simultaneous acquisition of brain metabolism images provided by ${ }^{18}$ F-FDG PET and morphological/functional images by MRI under the same clinical conditions. This new technique aimed to minimize the gap related to suboptimal co-registration when PET and MRI images were obtained separately on different machines. In addition, PET/MR decreased the radiation exposure compared with PET-CT, a notable advantage, especially in children. The role of PET/MR has been lately assessed in focal epilepsy and some studies have shown an increased its accuracy compared with PET-CT and MRI performed separately for the localisation of the epileptogenic zone (EZ) and the detection of structural lesions [14-18]. However, the additional value for the surgical decision-making of this new hybrid technique remains to be established.

We aimed to assess the role of PET/MR in a population of patients investigated for intractable focal epilepsy and candidates for surgery. We compared the results of PET/MR images with those obtained by a previous PET coregistered with MRI (PET + MR) in the same patients. The first analysis consisted in a systematic comparison of both imaging data including technical characteristics, sensitivity, and specificity. The second analysis aimed to determine the clinical impact of the PET/MR based on 1) the detection of new structural lesions compared with the reference images; 2 ) the potential change in decision-making and surgical management; 3 ) the results of the new surgical strategy. 
We hypothesized that the PET/MR may provide a better quality of co-registration and images, a higher degree of confidence for the visual analysis, an increased rate of lesion detection, and finally a higher number of patients referred for surgery and achieving a favorable outcome.

\section{Patients And Methods}

The study was conducted retrospectively in a single institution (SHFJ, CEA, Orsay, France) including patients referred for intractable focal epilepsy between 2017 and 2020.

\section{Inclusion criteria.}

1) All consecutive patients who underwent an ${ }^{18}$ F-FDG PET/MR at SHFJ for intractable focal epilepsy, having a previous

${ }^{18}$ F-FDG PET (in the same institution or not) available for review and MRI including 3D-T1 and FLAIR (Fluid Attenuation Inversion Recovery) sequences for the co-registration of PET and MRI; 2) Clinical data available (history of epilepsy, localisation of epileptic focus, result of surgery when performed); 3) Informed consent given by patients or parents for children. The study was approved by the local institutional review board (SHFJ-Codir 06-24-2019).

\section{Presurgical work-up and surgery.}

Presurgical evaluation was conducted in the Epilepsy Unit (Department of Neurosurgery, GHU Paris-Sainte-Anne), including video-EEG recordings, 3T morphological and functional MRI, and neuropsychological assessment for all patients. In addition, stereo-electroencephalography (SEEG) was performed in nine patients. SEEG electrode implantation was based on the integration of electroclinical and imaging data including PET results [19]. Cortical resection was performed using microsurgical techniques, after a multidisciplinary confrontation of the presurgical data. Histological analyses of the cortical specimen and classification of FCDs were based on international recommendations [20]. Radiofrequency thermocoagulations (RFTC) consisted in performing stereotactically coalescent thermal lesions to destroy a brain target as previously described [21]. Seizure-outcome assessment was based on Engel's classification [22].

\section{Imaging data.}

${ }^{18}$ F-FDG PET/MR. All acquisitions were performed on the SIGNA PET-MR machine (GE Healthcare, Milwaukee, Wisconsin, USA) based on a 3T 750w MR scanner, a whole-body MRI system with a $70 \mathrm{~cm}$ patient bore. The PET detector ring comprised 28 identical water-cooled modules, with a total of 20160 Lutetium-based scintillator (LBS) crystals (720 per module, crystal dimension: $3.95 \times 5.3 \times 25 \mathrm{~mm}^{3}$ ) read by arrays of Hamamatsu SiPM (Silicon PhotoMultiplier) devices (HPK S12044). Other main characteristics were as follows: PET bore diameter: $60 \mathrm{~cm}$, axial FOV: $25 \mathrm{~cm}$, sensitivity: $22.9 \mathrm{cps} / \mathrm{kBq}$, trans-axial spatial resolution: $4.3 \mathrm{~mm}$ at $1 \mathrm{~cm}$ [23]. Attenuation correction (AC) was based on fast zero-echo-time (ZTE) MRI derived from proton-density-weighted ZTE images by applying tissue segmentation and assigning continuous attenuation values to the bone [24]. ${ }^{18} \mathrm{~F}-\mathrm{FDG}$ was injected intravenously 30 min before image acquisition, at a mean dose of $3 \mathrm{MBq} / \mathrm{kg}$ of body-weight, in an awake and resting state, in a quiet, dimly lit environment, and carefully monitored for head movements and ictal events.

The MRI protocol was similar to the one used for patients investigated for drug-resistant epilepsy, including anatomical sequences in 3D gradient echo T1 (1 mm isotropic, $256 \times 256)$, coronal 2D T2 FSE ( $2.8 \mathrm{~mm}, 640 \times 448)$ in hippocampal plan for temporal lobe epilepsy (TLE) and AC-PC plan for extratemporal lobe epilepsy (ETLE), and 3D FLAIR (1.4 mm, $224 \times 224$ ) sequences as well as other sequences not used for the present study: 2D BOLD (blood oxygen level dependent) echo-planar imaging (EPI) and diffusion tensor imaging (DTI, 40 directories). The duration of the exam was $30 \mathrm{~min}$. 
Reference Imaging. For 14 patients, previous ${ }^{18} \mathrm{~F}$-FDG PET examination was performed in the same centre as the PET/MR (SHFJ), either on a PET-CT (Siemens Biograph 6) in 10 patients or on a 3D head-dedicated PET camera (Siemens Exact HR+) in four patients. For the 11 other patients, it was performed on different PET cameras, the characteristics of which are detailed in Table $1 .{ }^{18} \mathrm{~F}-\mathrm{FDG}$ was injected intravenously at a mean dose of $3 \mathrm{MBq} / \mathrm{kg}$ of body-weight in the same conditions as for the PET/MR. Image acquisition started $30 \mathrm{~min}$ after the injection and ended 15-20 min later.

Table 1

Technical characteristics of the different PET cameras used in the study.

\begin{tabular}{|c|c|c|c|c|c|}
\hline & $\begin{array}{l}\mathrm{PET} / \mathrm{MR} \text { GE N = } \\
25\end{array}$ & $\begin{array}{l}\text { PET-CT Siemens } N= \\
14 \\
\text { Biograph } 6 *(N=10) \\
\text { mCT** }(N=4)\end{array}$ & $\begin{array}{l}\text { PET-CT GE } \\
\text { Discovery } 670 \\
\mathrm{~N}=5\end{array}$ & $\begin{array}{l}\text { PET- } \\
\text { CT } \\
\text { Philips } \\
\text { TF } 64 \\
\mathrm{~N}=2\end{array}$ & $\begin{array}{l}\text { PET Siemens } \\
\text { Exact-HR+ } \\
\mathrm{N}=4\end{array}$ \\
\hline Year & 2015 & *2009/**2013 & 2010 & 2013 & 2002 \\
\hline $\begin{array}{l}\text { Attenuation } \\
\text { correction }\end{array}$ & ZTE & CT & CT & CT & ${ }^{68} \mathrm{Ge} 3$ line source \\
\hline Reconstruction & $\begin{array}{l}\text { 3D TOF OSEM } \\
8 \text { iterations (i) } \\
28 \text { subsets (s) }\end{array}$ & $\begin{array}{l}\text { *OSEM 3D }(\mathrm{N}=5) \\
\text { *TrueX 6i 16s }(\mathrm{N}=5) \\
\text { **PSF + TOF 8i 21s }\end{array}$ & VPFXS + Sharp IR & $\begin{array}{l}\text { BLOB- } \\
\text { OS-TF }\end{array}$ & $\begin{array}{l}\text { Back-projection } \\
\text { Hann filter } 4.0 \mathrm{~mm}\end{array}$ \\
\hline $\begin{array}{l}\text { Axial spatial } \\
\text { resolution }\end{array}$ & $4.3 \mathrm{~mm}$ & $\star 4.5 \mathrm{~mm} * \star 4.4 \mathrm{~mm}$ & $4.7 \mathrm{~mm}$ & $\begin{array}{l}4.8 \\
\mathrm{~mm}\end{array}$ & $4.2 \mathrm{~mm}$ \\
\hline Voxel size & $\begin{array}{l}1.578 \times 1.578 \times 2.78 \\
\mathrm{~mm}\end{array}$ & $\begin{array}{l}\text { *1.018x1.018x1.5 } \\
\mathrm{mm} \\
\star \star 1.018 \times 1.018 \times 2.027 \\
\mathrm{~mm}\end{array}$ & $\begin{array}{l}1.172 \times 1.172 \times 3.27 \\
\mathrm{~mm}\end{array}$ & $\begin{array}{l}2 \times 2 \times 2 \\
\mathrm{~mm}\end{array}$ & $\begin{array}{l}2.425 \times 2.425 \times 2.425 \\
\mathrm{~mm}\end{array}$ \\
\hline
\end{tabular}

The MRI used for the co-registration with the PET were performed on a 3 Tesla MRI (Discovery MR750; General Electric Healthcare) in 8 patients. In the other patients, it was performed on different machines with a magnetic field of 3 Tesla (20 patients) or 1.5 Tesla (5 patients), at the same time as the PET. The MRI protocol included 3D T1-weighted $1.2 \mathrm{~mm}$ thick contiguous slices, and 3D FLAIR sequences in 20 patients (2D FLAIR in 5 patients).

\section{Anonymisation of the data}

Data was de-identified using the Dicom dedicated tool from GE Healthcare. A random number was generated for each subject imaging session as last name, and "MRI" and "PET" as first name to separate both modalities from the PET/MR or PET and MRI examinations for separately acquired data.

Imaging analysis. Visual analysis of the PET/MR and PET + MR was performed by two readers on the working stations (Syngo.via version VA30 Siemens Healthcare and SIGNA PET/MR, GEMS): a junior nuclear medicine physician (reader 1, $\mathrm{AF}$ ) and a senior neuroradiologist with experience in PET and epilepsy imaging (reader 2, CM). They were informed of the initial clinical hypothesis, which was limited to the lobe and side of the epileptogenic focus but blinded of PET modality. The analysis was performed in 10 sessions of 5 different exams randomized for the type of acquisition in 
order to minimize the learning effect. Location and extent of metabolic abnormalities were classified as focal (involving a single gyrus), infralobar (involving several gyri in the same lobe), lobar or multilobar (involving the whole lobe or several lobes), using a semi-quantitative grading of the metabolic changes. The look-up table (LUT) divided the colour scale roughly equally, with upper scale normalization of $80 \%$ and background noise of $20 \%$, followed by a new upper scale normalization if necessary. Hypometabolism was classified as mild, moderate, and severe according to the percentage of SUV reduction $(10-15,15-20,>20)$, and doubtful if $<10 \%$. The MRI was simultaneously reviewed searching for cortical abnormalities concordant with the hypometabolic areas. Metabolic and structural abnormalities were localised according to their hemispheric, lobar and sub-lobar location, involving 64 sites as follow: 1) lateralisation on the right side, left side, or bilateral, symmetric or not; 2) temporal lobe (hippocampus, parahippocampal gyrus, amygdala, pole, anterior/posterior part of superior, middle, inferior gyrus); 3 ) frontal lobe (pole, orbitofrontal, anterior cingulate gyrus, mesial/dorsolateral part of prefrontal, premotor, precentral areas), 4) central region (mesial/dorsolateral part of the motor cortex, pre and post-central operculum); 5) anterior/posterior insula, 6) mesial/lateral part of parietal and occipital lobes. Subcortical structures (basal ganglia, thalamus, cerebellum) were also included in the analysis. For each file, the analysis grid included the self-assessment of the co-registration, quality of images and degree of confidence ( 1 = poor, 2 = acceptable, 3 = good), with a minimal score of 3 and a maximal score of 9 . In addition, the duration of the analysis was noted for each file. The PET was considered positive if an undoubtful focal area of hypometabolism was visually detected, whereas it was negative if no or only doubtful changes were found. The MRI was considered positive if demonstrating a clear-cut abnormality on T1, FLAIR or both sequences, it was negative if no or doubtful signal changes were observed. Concordance between the readers was based on the positivity or negativity of the PET, and the location of metabolic abnormalities. It was considered as fully concordant if all results were similar, partially concordant if only minor differences were reported (i.e., precise location or extent of metabolic changes within the same lobe), and discordant in the other cases. A final review was performed for discordant results.

Comparison between the PET/MR and PET + MR was performed for each reader by pooling the sum of different scores, the location and extend of metabolic changes, and the detection of a structural lesion on the MRI. The PET/MR was considered better performing than the PET + MR if the sum of scores increased of at least 2 points, and/or if negative findings changed to positive findings (either on the PET, the MRI or both). Sensitivity and specificity were assessed on the basis of clinical data obtained at the last evaluation, including the results of SEEG, surgical outcome and histological findings, according to the following formulation: - Sensitivity = True Positive (TP) / True Positive (TP) + False Negative (FN) - Specificity = True Negative (TN) /True Negative (TN) + False Positive (FP). In a separate analysis (FC), the role of the PET/MR was retrospectively assessed by comparing the surgical indication and strategy before and after performing the PET/MR, including the need of invasive monitoring (SEEG), the planning of electrode implantation and the surgical outcome.

Statistical analyses. For interobserver agreement, only PET positivity or negativity (from the PET/MR and PET standalone) were considered. The concordance analysis was conducted using the kappa index, classifying concordance as slight $(0.00-0.20)$, fair $(0.21-0.40)$, moderate $(0.41-0.60)$, substantial $(0.61-0.80)$, and almost perfect $(0.81-1.00)$.

\section{Results}

\section{Patient population.}

The studied population consisted in 25 patients (16 females), aged from 13 to 60 years (mean: 26 years, SD:12). Epilepsy onset ranged from 2 to 35 years (mean: 10 years, SD: 7.1) and duration of epilepsy from 4 to 36 years (mean: 16 years, SD: 9.1). Epilepsy was drug-resistant in all patients, with a high seizure frequency in most cases (daily in 16, weekly or monthly in the others). The treatment consisted of the association of two antiepileptic drugs (AED) for 14 
patients and of three AED for 11 others. The presumed location of the epileptic focus was temporal (9 cases), frontal (9 cases), central (6 cases) and parietal (1 case). The lateralisation was on the right hemisphere in 10 cases, on the left in 13 cases and undetermined in 2 . The MRI was initially considered negative in 21 patients and positive in the four others (hippocampal sclerosis in three and FCD in one).

\section{PET/MR and PET + MR acquisition}

The delay between the PET/MR and the previous PET ranged from 0 to 10 years (mean: 2.9, SD: 2.3). In two patients, the PET/MR was performed immediately after the PET-CT (and 45 min after ${ }^{18} \mathrm{~F}$-FDG injection), to assess potential differences between the two exams. For the other patients, the reason for performing the PET/MR was a lack of sufficient information provided by the previous PET + MR (16 patients), a long delay from the initial PET before reconsidering surgery ( 2 cases), a widespread hypometabolism possibly related to a post-ictal state ( 3 cases), and the confirmation of a doubtful result (2 cases). No major change in terms of seizure frequency and AEDs was reported between this time interval, despite some modification for eight of them (replacement of one AED or introduction a new one). The delay between the last seizure and the PET/MR ranged from 0 to 30 days (mean: 7.2, SD: 10.2). Nine patients had one or several seizures during the night preceding the PET/MR but occurring less than 6 hours before the ${ }^{18}$ F-FDG injection in only one of them. In one of these patients, a seizure occurred 54 min after injection, 22 min after the onset of image acquisition which was stopped. The delay between the last seizure and the previous PET ranged from 0 to 75 days in 12 patients (mean 15.5, SD: 22.2) and was unknown in 13 patients. Five patients had one or several seizures during the precedent night, but none occurring less than six hours before injection. One patient had a short seizure 20 min after injection. Another patient suffered from diabetes with relatively high glycemia (1.6g/l) before the two exams, resulting in poor quality of images.

$\mathrm{PET} / \mathrm{MR}$ versus PET + MR analysis (Table 2) 
Table 2

Comparison between PET/MR and PET + MR in the patient population $(\mathrm{N}=25)$.

\begin{tabular}{|c|c|c|c|c|c|c|}
\hline & $\begin{array}{l}\text { PET + MR } \\
1\end{array}$ & PET/MR 1 & $\begin{array}{l}\mathrm{PET}+\mathrm{MR} \\
2\end{array}$ & PET/MR 2 & $\begin{array}{l}\mathrm{PET}+\mathrm{MR}(1+ \\
\text { 2) }\end{array}$ & $\begin{array}{l}\mathrm{PET} / \mathrm{MR}(1+ \\
\text { 2) }\end{array}$ \\
\hline PET positive/negative & $14 / 11$ & $18 / 7$ & $19 / 6$ & $22 / 3$ & $19 / 6$ & $22 / 3$ \\
\hline Main localisation & $7 / 3 / 4 / 0 / 0$ & $9 / 5 / 4 / 0 / 0$ & $7 / 2 / 8 / 1 / 1$ & $8 / 4 / 8 / 1 / 1$ & $7 / 3 / 7 / 1 / 1$ & $8 / 5 / 7 / 1 / 1$ \\
\hline \multicolumn{7}{|l|}{$\mathrm{T} / \mathrm{F} / \mathrm{C} / \mathrm{P} / \mathrm{I}$} \\
\hline Extent G/IL/L/ML & $5 / 8 / 1 / 0$ & $8 / 5 / 1 / 4$ & 10/7/1/1 & $13 / 6 / 2 / 1$ & $10 / 7 / 1 / 1$ & $13 / 6 / 2 / 1$ \\
\hline MRI positive/negative & $4 / 21$ & $8 / 17$ & $10 / 15$ & $14 / 11$ & $8 / 17$ & $14 / 11$ \\
\hline $\begin{array}{l}\text { Score } 1 \text { (co- } \\
\text { registration) }\end{array}$ & 49 & 67 & 54 & 71 & 51.5 & 69 \\
\hline Score 2 (quality) & 36 & 61 & 53 & 63 & 44.5 & 62 \\
\hline Score 3 (confidence) & 46 & 58 & 55 & 62 & 50.5 & 60 \\
\hline $\begin{array}{l}\text { Duration of analysis } \\
(\mathrm{mn})\end{array}$ & $9-20(14)$ & $9-20(14)$ & $6-34(15)$ & $5-30(15)$ & & \\
\hline $\mathrm{PET} / \mathrm{MR}>\mathrm{PET}+\mathrm{MR}$ & & 14 & & 14 & & 14 \\
\hline $\mathrm{PET} / \mathrm{MR}<\mathrm{PET}+\mathrm{MR}$ & 1 & & 4 & & 3 & \\
\hline Sensitivity & $\begin{array}{l}13 / 22 \\
(59 \%)\end{array}$ & $\begin{array}{l}16 / 21 \\
(76 \%)\end{array}$ & $\begin{array}{l}18 / 22 \\
(82 \%)\end{array}$ & $\begin{array}{l}20 / 21 \\
(95 \%)\end{array}$ & 19/23 (83\%) & $22 / 23(96 \%)$ \\
\hline \multirow[t]{3}{*}{ Specificity } & $2 / 3(67 \%)$ & $2 / 4(50 \%)$ & $2 / 3(67 \%)$ & $2 / 4(50 \%)$ & $2 / 2(100 \%)$ & $2 / 2(100 \%)$ \\
\hline & TP 13 FP 1 & TP 16 FP 2 & TP 18 FP 1 & TP 20 FP 2 & TP 19 FP 0 & TP 22 FP 0 \\
\hline & FN 9 TN 2 & FN 5 TN 2 & FN 4 TN 2 & FN 1 TN 2 & FN 4 TN 2 & FN 1 TN 2 \\
\hline \multicolumn{7}{|c|}{$1=$ reader $1 ; 2=$ reader $2 ; 1+2=$ after consensus } \\
\hline \multicolumn{7}{|c|}{ T = Temporal; F = Frontal; C = Central; P = Parietal; I = Insula } \\
\hline \multicolumn{7}{|l|}{ Sensitivity TP/ TP + FN } \\
\hline Specificity TN/TN + FP & & & & & & \\
\hline
\end{tabular}

Interobserver concordance rate was of $68 \%$ for the PET + MR and $80 \%$ for the PET/MR. The kappa index was 0.403 for the PET + MR $(p=0.026)$ and $0.233(p=0.069)$ for the PET/MR. These relatively low indexes were explained by the few numbers of negative exams. The main discrepancies consisted in the detection and location of hypometabolism, whereas a partial concordance was mainly observed for the analysis of MRI, which was related to the training and experience of the readers. The duration of analysis was similar for the two readers (around $15 \mathrm{~min}$ ).

The PET/MR obtained higher scores than the PET + MR for each item and for the two readers. The best improvement was noted for the quality of images (reader 1) and the co-registration (reader 2), while a higher degree of confidence was obtained for both readers. Considering the sum of scores and the change of category (PET and/or MR negative becoming positive), the PET/MR was stated better performing than the PET + MR in 14 cases (56\%) for both readers (Fig. 1), whereas the PET + MR was considered better in one case by reader 1 and four cases for reader 2 (mainly due to the poor quality of the MRI due to motion artefacts). Notably, the delay between both exams was similar whether the $\mathrm{PET} / \mathrm{MR}$ was considered better than the PET + MR or not (mean: 3 years each). The extent and location of 
hypometabolism was somewhat different for the two readers with a better detection in difficult regions (central, parietal and insula) and small areas of hypometabolism for reader 2, traducing the role of experience for the results of analysis.

Interestingly, the PET/MR allowed the detection of new structural lesions by the two readers. For reader 1, the positive MRI rate increased from four to eight (16-32\%). For reader 2, this rate was high in two modalities (10 positive MRI after the co-registration with PET contrasting with four for MRI stand-alone), however the PET/MR allowed the detection of four additional structural lesions, corresponding to FCD type 2 proven by the histological examination in three of them (Fig. 2). Finally, the sensitivity was higher for the PET/MR compared with the PET + MR for both readers (76\% versus $59 \%$ respectively for the reader $1 ; 95 \%$ versus $86 \%$ for the reader 2 ). However, the specificity was slightly lower due to few true negative findings. After consensus, the sensitivity reached $96 \%$ and the specificity $100 \%$.

Clinical impact of the PET/MR (Table 3).

Table 3

Role of PET/MR on the surgical management of patients $(\mathrm{N}=25)$.

\begin{tabular}{|c|c|c|}
\hline & PET + MR & PET/MR \\
\hline PET positive & $19(76 \%)$ & $23(92 \%)$ \\
\hline MR positive & $8(32 \%)$ & $14(56 \%)$ \\
\hline Surgical management & 2 & 2 \\
\hline Surgery not indicated & $0 / 17$ & $9 / 2$ \\
\hline SEEG (performed/planned) & $0 / 4$ & $14 / 3$ \\
\hline Corticectomy (performed/planned) & & $5^{\star}$ \\
\hline \multicolumn{3}{|l|}{ RFTC } \\
\hline Histology $(n=14)$ & & 5 \\
\hline FCD2A & & 3 \\
\hline FCD2B & & 2 \\
\hline HS & & 4 \\
\hline \multicolumn{3}{|l|}{ Others** } \\
\hline Seizure outcome *** & & $13 / 16(81 \%)$ \\
\hline Engel class I, all patients & & $12 / 13(92 \%)$ \\
\hline Corticectomy & & $1 / 3(33 \%)$ \\
\hline \multicolumn{3}{|l|}{ RFTC } \\
\hline \multicolumn{3}{|c|}{ RFTC = Radio-Frequency Thermo-Coagulations; FCD = Focal cortical dysplasia; HS = Hippocampal Sclerosis } \\
\hline \multicolumn{3}{|l|}{ *1 patient had corticectomy and RFTC } \\
\hline \multicolumn{3}{|c|}{ ** Focal neuronal lipofuscinosis 1, no specific lesion 3} \\
\hline ***For patients having at least $6 \mathrm{~mol}$ & & \\
\hline
\end{tabular}

All patients of this series were candidates for surgery, but for most of them invasive monitoring (SEEG) was discussed on the basis of the classical presurgical work-up. In addition, visual assessment of the PET was considered negative in six patients, and the MRI was negative in 21 patients. After co-registration of the PET and MRI, four new structural 
lesions were identified. After the PET/MR, only two PET exams remain negative and six additional new lesions were detected on the MRI (24\%). Considering the data provided by the PET/MR, the initial planning of SEEG in 17 patients was reduced to 11. Among the six patients in whom the invasive monitoring was avoided, five had a subtle structural lesion found on the PET/MR, corresponding to FCD type 2 confirmed by histology, while no specific lesion was found in the latter patient. All patients operated on without SEEG achieved a favorable outcome after the cortical resection. For the 11 other patients where the SEEG remained indicated, the planning of electrode implantation was modified in four of them. Therefore, the change of surgical strategy was substantial for 10 patients (40\%). Of note, surgery was not indicated in only two patients, due to a benefit/risk ratio considered too low for this counselling. For the other patients, most of those who underwent a corticectomy achieved a seizure-free outcome (Engel class I in 12/13 cases (92\%), with a minimal 6-month follow-up (up to 4 years, mean 1.5). In addition, one patient was also seizure-free after the RFTC.

\section{Discussion}

In this study, we demonstrate the additional value of the PET/MR and its influence on the surgical management of epileptic patients examined for intractable focal epilepsy. Based on a series of 25 patients with well-defined EZ and successful surgery for most of them, we can precisely assess the role of the new hybrid PET/MR compared to the reference imaging. We emphasized that the studied population accounted for the most difficult patients in epilepsy surgery including a high rate of extratemporal location and initially negative MRI cases.

First of all, we show that the PET/MR offers better accuracy than the PET + MR, in terms of co-registration and quality of images, and therefore a higher degree of confidence for the reader, whatever his/her training and experience. Notably the sensitivity was increased of $13-17 \%$, despite a slight decrease of specificity due to the small number of negative studies. However, the specificity was improved after consensus. Previous studies have established the equivalent properties of the PET/MR compared with the PET-CT by visual or quantified analysis [16, 25-27]. Other studies have shown the higher sensitivity of the PET/MR to detect epileptogenic lesions compared with PET and MRI stand-alone [15, 17-18], but to our knowledge, the additional value of the PET/MR compared with the PET + MR has not been demonstrated to date.

Importantly, we found that the PET/MR allowed the detection of structural lesions in a high proportion of patients (24 \%) in whom the MRI was initially considered negative, despite optimal examination including the co-registration with the PET. This multimodal approach has been previously reported as increasing the detection of FCDs [8-11] and considered as the reference method that should be recommended as a standard procedure for MRI negative ETLE [12]. However, according to our data, the PET/MR reaches a new step for the detection of such epileptogenic lesions compared to the reference imaging. Reasons for this improvement may be related to 1) a better co-registration of the PET and MRI provided by the PET/MR; 2) a better detection of small lesions due to the simultaneous acquisition of high-resolution 3D-T1 MRI sequences (especially when located at the bottom of the sulcus, that is frequent for small FCDs); 3 ) a better analysis of FLAIR images guided by PET findings. Despite advances in fusion software, it may be difficult to generate optimal co-registration of PET and MR images acquired separately on different machines, at different times and under different conditions, including post-ictal state. In addition, visual analysis of 3D-T1 and FLAIR images is greatly facilitated by the simultaneous acquisition of the PET. It should also be notified that in healthy volunteers, the time of day may influence the brain morphometric measures, including gray matter, white matter and cerebrospinal fluid, with a highly significant reduction (more than one percent per $12 \mathrm{hr}$ day) in apparent brain volume, from morning to evening [28]. Moreover, these variations were found predominantly in frontal and temporal lobes, which are frequently involved in epileptic patients. This observation argues for the simultaneous acquisition of PET and MRI for a better accuracy of the co-registration, especially when searching for millimetric FCDs. 
Worthy of note, despite initial concerns about the correction of attenuation with PET/MR, equivalent properties with PETCT have been demonstrated afterward $[16,26]$. Interestingly, using ZTE-AC, the overall ${ }^{18} \mathrm{~F}-\mathrm{FDG}$ uptake bias has proved to be $25 \%$ lower than that of atlas-AC [24]. This progress was achieved by the improved registration and assignment of correct attenuation values for the skull. Especially in central structures, insula and cingulate areas, a significant improvement with ZTE-AC was found in comparison to clinical atlas-AC. This could be particularly relevant in our patient population in which such location was frequently involved. On the other hand, ZTE-AC was found to misclassify some of the nasal and mastoid areas as bone, which caused overestimation of ${ }^{18} \mathrm{~F}$-FDG uptake in the temporal lobe and cerebellum [24]. However, such inconvenience was of less importance since temporal hypometabolism is usually easy to detect, and a potential overestimation is unlikely to provide a false diagnosis.

Another potentially important advantage of the PET/MR is to compare the metabolic activity with other functional MR images such Arterial Spin Labeling (ASL), that could be highly influenced by the physiologic and pathologic conditions, especially the interictal and post-ictal states. This comparison was not tested in the present study but good correlations between the two modalities have been reported, providing complementary information in determining the EZ [25, 29].

A last (but not least) point is the one stop-shop concept of the hybrid PET/MR, which is more convenient for patients and clinicians. Indeed, for the patient, there is only one scan, which is more comfortable and less stressful, especially for children and patients with high seizure frequency. For the clinician, it is also more convenient to obtain both high quality PET and MR for a simultaneous reading.

Finally, considering the new information provided by the PET/MR in this study, the surgical management was modified in $40 \%$ of the patients. Invasive monitoring (SEEG) was avoided in six patients, without loss of chance since all were seizure-free after the cortical resection. Furthermore, all patients having FCD type 2 proven by histology (8 cases) were operated on without SEEG and achieved a favorable outcome. These results confirm those previously reported by our team in patients operated on for FCDs $[9,11]$ and support the view that SEEG may be avoided on the basis of PET/MR findings. For the patients in whom SEEG remained indicated, the planning of electrode implantation was modified in a third of the cases, allowing a better definition of the EZ. Overall, the PET/MR was considered to improve the surgical decision-making and outcome in nearly half of the patients.

Interestingly, the utility of the ${ }^{18}$ F-FDG PET alone was previously estimated at $53 \%$ in a large series of epileptic patients investigated for surgery [30]. In addition, improvement of seizure-free outcome has been observed after changing the imaging protocol including the PET in a pediatric population [31]. Moreover, the PET/MR was reported as providing better accuracy of electrode implantation during SEEG [32]. It can be suggested that the PET/MR may still increase the positive impact already provided by the PET alone in epilepsy surgery, as demonstrated in our population.

The final goal of a new technique is to prove its value for improving diagnosis and curative treatments for patients. Considering epilepsy surgery, seizure outcome and long-term results including stopping the antiepileptic medication are highly dependent on finding an epileptogenic lesion in the cortical specimen removed during the operation [33]. In ETLE and negative MRI cases (so called "non lesional epilepsy"), seizure-free outcome is commonly reported lower than in TLE and well-identified lesion on MRI. It was reported ranging from 15-38\% in previous large series of non-lesional ETLE in which the role of PET was not considered [34-36] and $27-46 \%$ in a previous meta-analysis [37]. In addition, non-lesional epilepsy was associated with a two to three times lower rate of seizure-free outcome than lesional epilepsy [38]. Contrasting with these results, the chance of being seizure-free increased to $67 \%$ when FCD type 2 was found by histological examination in the cortical specimen, as demonstrated in a recent large multicentric study [33]. This dramatic improvement of outcome highlights the role of imaging in identifying such epileptogenic lesion preoperatively. Accordingly, we found a high rate of seizure-free outcome in a previous cohort of patients operated on for intractable epilepsy due to FCD type 2. Based on a presurgical work-up including ${ }^{18}$ F-FDG PET in all cases, we did not find 
significant difference between MRI-positive and MRI-negative cases (Engel class I in $94 \%$ and $88 \%$ respectively) [4]. Further improvement should be expected with the PET/MR as suggested by the present series, including more successful surgery performed without SEEG. Notably, the better sensitivity of this hybrid imaging leads to revisit the proportion of truly non-lesional epilepsy patients that could be referred for surgery, questioning on the presence of a structural abnormality at the microscopic level.

\section{LIMITATIONS}

The first limitation of this study is the relatively small size of population, due to the difficulties for obtaining images in both modalities and sufficient clinical information to perform meaningful correlations. However, the well-localised EZ and the high rate of patients with favorable outcome after surgery enhance the confidence in our results. Secondly, the PET from the PET/MR provided optimised images compared to the previous PET, which were performed on different cameras with various spatial resolution and reconstruction methods. Nevertheless, it cannot explain the increase of sensitivity on its own, since a better co-registration was noted as frequently as a better quality of images by the two readers. In addition, the structural lesions newly detected on MRI cannot be attributed to a better quality of MRI or PET images independently but are rather due to the improvement of the fusion of both. Finally, the delay between the two examinations may play or role for the discrepancy, however we did not find any relationship between this delay and the improvement provided by the PET/MR. Furthermore, there was no major clinical change in terms of seizure frequency, AEDs and delay between the last seizure and the ${ }^{18} \mathrm{~F}-\mathrm{FDG}$ injection. It should be emphasized that optimal comparative studies are rare, other studies based on consecutive examinations having also some limitations related to the different delay after the ${ }^{18} \mathrm{~F}-\mathrm{FDG}$ injection. Furthermore, the main limitations in previous studies consisted in the variable (and sometimes weak) assessment of the clinical usefulness, that we believe having demonstrated in our study.

\section{Conclusion}

We demonstrated the clinical utility of the PET/MR in a series of patients investigated for focal drug-resistant epilepsy, in whom the simultaneous acquisition of the PET and MRI improved the detection of epileptogenic lesions, allowing a higher proportion of successful surgery and simplifying the presurgical work-up, even in the more complex cases. Combining "the best of two worlds", we postulate that these two imaging modalities acquired in a single session on a hybrid system would be helpful for the pre-surgical evaluation of epileptic patients and recommended in case of previous negative imaging findings.

\section{Declarations}

\section{AKNOWLEDGEMENTS}

The authors thank Claude Comtat for his methodological advices and all the SHFJ team, especially Philippe Gervais, Thierry Le Kieffre, and Christine Baron for carrying out the PET and PET/MR. They also thank Dr Catherine Chiron who performed the PET in children and the Sainte-Anne's team (Elisabeth Landré and Bertrand Devaux) for the patient care.

\section{COMPLIANCE WITH ETHICAL STANDARDS}

Funding: This work was performed on a platform member of France Life Imaging network (grant ANR-11-INBS-0006).

Conflicts of interest/Competing interests: Anthime Flaus declares that he has no conflict of interest; Charles Mellerio Flaus declares that he has no conflict of interest; Sebastian Rodrigo declares that he has no conflict of interest; Vincent Brulon declares that he has no conflict of interest: Vincent Lebon declares that he has no conflict of interest; Francine Chassoux declares that she has no conflict of interest.

Page $11 / 15$ 
Availability of data and material (data transparency) at SHFJ, CEA, Orsay France

Ethical approval: All procedures performed in studies involving human participants were in accordance with the ethical standards of the institutional and/or national research committee and with the 1964 Helsinki declaration and its later amendments or comparable ethical standards. Informed consent was obtained from all individual participants (and parents for children) included in the study. The study was approved by the local institutional review board (SHFJ-Codir 06-24-2019).

\section{References}

1. - Blumcke I, Spreafico R, Haaker G, Coras R, Kobow K, Bien CG, et al. Histopathological findings in brain tissue obtained during epilepsy surgery. N Engl J Med. 2017;377:1648-56.

2. - Ryvlin P, Cross JH, Rheims S. Epilepsy surgery in children and adults. Lancet Neurol. 2014;13:1114-26.

3. - Guerrini R, Duchowny M, Jayakar P, Krsek P, Kahane P, Tassi L, et al. Diagnostic methods and treatment options for focal cortical dysplasia. Epilepsia. 2015;56:1669-86.

4. - Chassoux F, Landre E, Mellerio C, Turak B, Mann MW, Daumas-Duport C, et al. Type II focal cortical dysplasia: Electro-clinical phenotypes and surgical outcome related to imaging. Epilepsia. 2012;53:349-58.

5. - Mellerio C, Labeyrie MA, Chassoux F, Daumas-Duport C, Landre E, Turak B, et al. Optimizing MR imaging detection of type 2 focal cortical dysplasia: best criteria for clinical practice. AJNR Am J Neuroradiol. 2012;33:1932-8.

6. - Mellerio C, Labeyrie MA, Chassoux F, Roca P, Alami O, Plat M, et al. 3T MRI improves the detection of transmantle sign in type 2 focal cortical dysplasia. Epilepsia. 2014;55:117-22.

7. - Bernasconi A, Cendes F, Theodore WH, Gill RS, Koepp MJ, Hogan RE, et al. Recommendations for the use of structural magnetic resonance imaging in the care of patients with epilepsy: A consensus report from the International League Against Epilepsy Neuroimaging Task Force. Epilepsia. 2019;60:1054-68.

8. - Salamon N, Kung J, Shaw SJ, Koo J, Koh S, Wu JY, et al. FDG-PET/MRI coregistration improves detection of cortical dysplasia in patients with epilepsy. Neurology. 2008;71:1594-601.

9. - Chassoux F, Rodrigo S, Semah F, Beuvon F, Landré E, Devaux B, et al. FDG-PET improves surgical outcome in negative-MRI Taylor type focal cortical dysplasias. Neurology. 2010;75:2168-75.

10. - Rubí S, Setoain X, Donaire A, Bargalló N, Sanmartí F, Carreño M, et al. Validation of FDG-PET/MRI coregistration in nonlesional refractory childhood epilepsy. Epilepsia. 2011;52:2216-24.

11. - Desarnaud S, Mellerio C, Semah F, Laurent A, Landre E, Devaux B, Chiron C, Lebon V, Chassoux F. 18F-FDG PET in drug-resistant epilepsy due to focal cortical dysplasia type 2: additional value of electroclinical data and coregistration with MRI. Eur J Nucl Med Mol Imaging. 2018;45:1449-60.

12. - Ding Y, Zhu Y, Jiang B, Zhou Y, Jin B, Hou H, et al. 18F-FDG PET and high-resolution MRI co-registration for presurgical evaluation of patients with conventional MRI-negative refractory extra-temporal lobe epilepsy. Eur $\mathrm{J}$ Nucl Med Mol Imaging. 2018;45:1567-72.

13. - Tan YL, Kim H, Lee S, Tihan T, Ver Hoef L, Mueller SG, et al. Quantitative surface analysis of combined MRI and PET enhances detection of focal cortical dysplasias. Neurolmage. 2018;166:10-8.

14. - Ding YS, Chen BB, Glielmi C, Friedman K, Devinsky O. A pilot study in epilepsy patients using simultaneous PET/MR. Am J Nucl Med Mol Imaging. 2014;4:459-70.

15. - Shin HW, Jewells V, Sheikh A, Zhang J, Zhu H, An H, et al. Initial experience in hybrid PET-MRI for evaluation of refractory focal onset epilepsy. Seizure. 2015;31:1-4.

16. - Paldino MJ, Yang E, Jones JY, Mahmood N, Sher A, Zhang W. Comparison of the diagnostic accuracy of PET/MRI to PET/CT-acquired FDG brain exams for seizure focus detection: a prospective study. Pediatr Radiol.

Page 12/15 
2017;47:1500-7.

17. - Oldan JD, Shin HW, Khandani AH, Zamora C, Benefield T, Jewells V. Subsequent experience in hybrid PET-MRI for evaluation of refractory focal onset epilepsy. Seizure: European Journal of Epilepsy. 2018;61:128-34.

18. - Kikuchi K, Togao O, Yamashita K, Momosaka D, Nakayama T, Kitamura Y, et al. Diagnostic accuracy for the epileptogenic zone detection in focal epilepsy could be higher in FDG-PET/MRI than in FDG-PET/CT. Eur Radiol. 2020 Oct 15. doi:10.1007/s00330-020-07389-1.

19. - Chassoux F, Navarro V, Catenoix H, Valton L, Vignal JP. Planning and management of SEEG. Neurophysiol Clin. 2018;48:25-37.

20. - Blümcke I, Thom M, Aronica E, Armstrong DD, Vinters HV, Palmini A, et al. The clinicopathologic spectrum of focal cortical dysplasias: a consensus classification proposed by an ad hoc Task Force of the ILAE Diagnostic Methods Commission. Epilepsia. 2011;52:158-74.

21. - Mullatti N, Landre E, Mellerio C, Oliveira AJ, Laurent A, Turak B, Devaux B, Chassoux F. Stereotactic thermocoagulation for insular epilepsy: Lessons from successes and failures. Epilepsia. 2019;60:1565-79.

22. -Engel J Jr, Van Ness PC, Rasmussen TB, Ojemann LM. Outcome with respect to epileptic seizures. Engel JJr. Surgical treatment of the epilepsies. 2nd ed. Raven Press, New York, 1993:pp. 609-21.

23. - Levin CS, Maramraju SH, Khalighi MM, Deller TW, Delso G, Jansen F. Design features and mutual compatibility studies of the time-of-flight PET capable GE SIGNA PET/MR System. IEEE Trans Med Imaging. 2016;35:1907-14.

24. -Sekine T, Voert EGWter, Warnock G, Buck A, Huellner M, Veit-Haibach P, et al. Clinical evaluation of zero-echo-time attenuation correction for brain 18F-FDG PET/MRI: comparison with atlas attenuation correction. J Nucl Med. 2016;57:1927-32.

25. - Boscolo Galazzo I, Mattoli MV, Pizzini FB, De Vita E, Barnes A, Duncan JS, et al. Cerebral metabolism and perfusion in MR-negative individuals with refractory focal epilepsy assessed by simultaneous acquisition of $18 \mathrm{~F}-$ FDG PET and arterial spin labeling. Neurolmage Clinical. 2016;11648-57.

26. - Ladefogeda CN, Lawa I, Anazodob U, Izquierdo-Garciac StLK, Catanac D. C, et al. A multi-centre evaluation of eleven clinically feasible brain PET/MRI attenuation correction techniques using a large cohort of patients. Neurolmage. 2017;147:346-59.

27. - Traub-Weidinger T, Muzik O, Sundar LKS, Aull-Watschinger S, Beyer T, Hacker M, et al. Utility of absolute quantification in non-lesional extratemporal lobe epilepsy using FDG PET/MR Imaging. Front Neurol. 2020;11:54. doi:10.3389/fneur.2020.00054.

28. -Trefler A, Sadeghi N, Thomas AG, Pierpaoli C, Bakera CL, Thomas C. Impact of time-of-day on brain morphometric measures derived from T1-weighted magnetic resonance imaging. Neuroimage. 2016;133:41-52.

29. - Shang K, Wang J, Fan X, Cui B, Ma J, Yang H, et al. Clinical value of hybrid TOF-PET/MR imaging-based multiparametric imaging in localizing seizure focus in patients with MRI-negative temporal lobe epilepsy. AJNR Am J Neuroradiol. 2018;39:1791-98.

30. - Rathore C, Dickson JC, Teotónio R, Ell P, Duncan JS. The utility of 18F-fluorodeoxyglucose PET (FDG PET) in epilepsy surgery. Epilepsy Res. 2014;108:1306-14.

31. - Rubinger L, Chan C, D'Arco F, Moineddin R, Muthaffar O, Rutka JT, et al. Change in presurgical diagnostic imaging evaluation affects subsequent pediatric epilepsy surgery outcome. Epilepsia. 2016;57:32-40.

32. - Zhang M, Liu W, Huang P, Lin X, Huang X, Meng H, et al. Utility of hybrid PET/MRI multiparametric imaging in navigating SEEG placement in refractory epilepsy. Seizure: European Journal of Epilepsy. 2020;81:295-303.

33. - Lamberink HJ, Otte WM, Blümcke I, Braun KPJ, on behalf of the European Epilepsy Brain Bank writing group, study group, and the European Reference Network EpiCARE. Seizure outcome and use of antiepileptic drugs after epilepsy

Page $13 / 15$ 
surgery according to histopathological diagnosis: a retrospective multicentre cohort study. Lancet Neurol. 2020;19:748-57.

34. - McIntosh AM, Averill CA, Kalnins RM, Mitchell LA, Fabinyi GCA, Jackson GD, et al. Long-term seizure outcome and risk factors for recurrence after extratemporal epilepsy surgery. Epilepsia. 2012;53:970-8.

35. - Noe K, Sulc V, Wong-Kisiel L, Wirrell E, Van Gompel JJ, Wetjen N, et al. Long-term outcomes after nonlesional extratemporal lobe epilepsy surgery. JAMA Neurol. 2013;70:1003-8.

36. - Bell GS, de Tisi J, Gonzalez-Fraile JC, Peacock JL, McEvoy AW, Harkness WFJ, et al. Factors affecting seizure outcome after epilepsy surgery: an observational series. J Neurol Neurosurg Psychiatry. 2017;88:933-40.

37. - Tellez-Zenteno JF, Dhar R, Wiebe S. Long-term seizure outcomes following epilepsy surgery: a systematic review and meta-analysis. Brain. 2005;128:1188-98.

38. - Tellez-Zenteno JF, Hernandez Ronquillo L, Moien-Afshari F, Wiebe S. Surgical outcomes in lesional and nonlesional epilepsy: a systematic review and meta-analysis. Epilepsy Res. 2010;89:310-8.

\section{Figures}

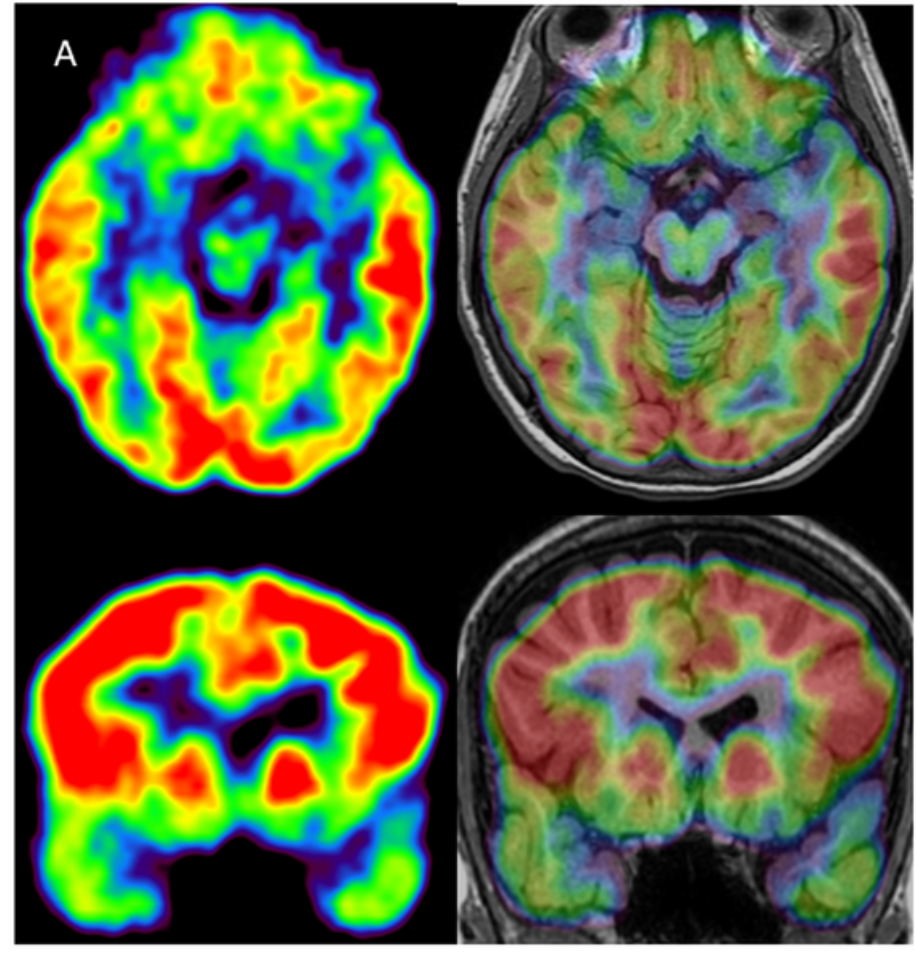

$\mathrm{PET}+\mathrm{MR}$

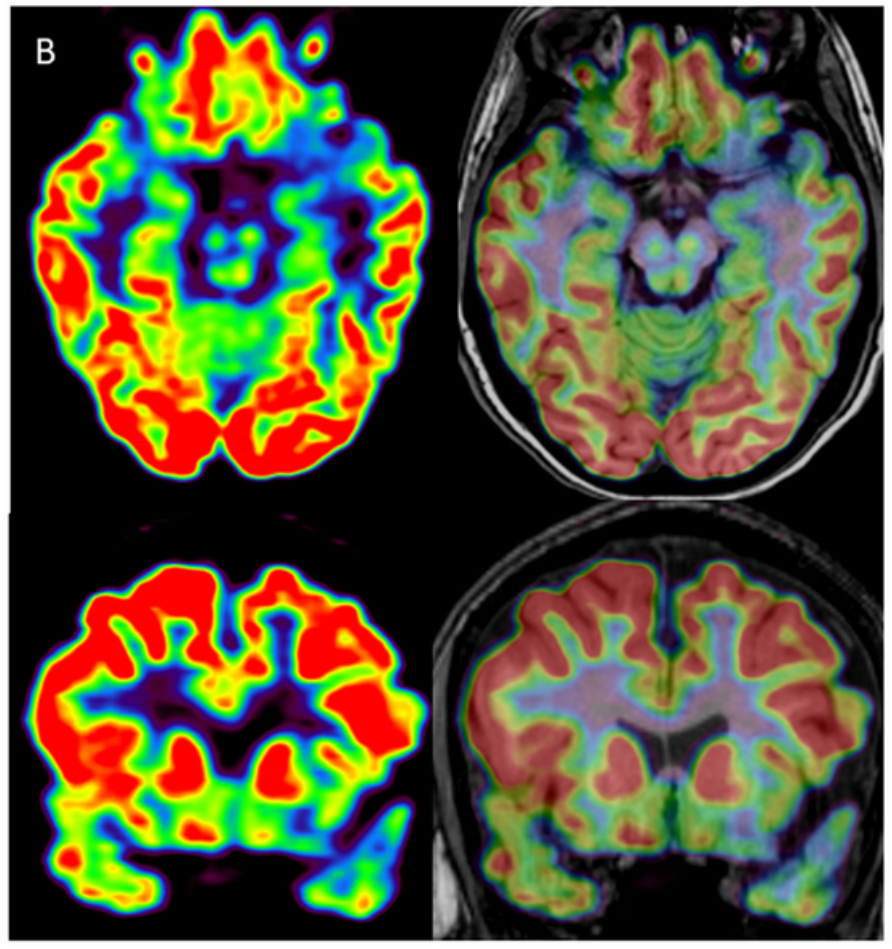

$\mathrm{PET} / \mathrm{MR}$

\section{Figure 1}

Comparison between PET+MR (A) and PET/MR (B); axial and coronal plane, PET alone and co-registered on MRI. 17 year-old female, drug resistant epilepsy since the age of 2 years. Nocturnal frontal seizures (3/night), left frontotemporal ictal onset, negative MRI (3Tesla). First PET examination co-registered on MRI at 11 years considered negative; PET/MR 6 years later, showing a focal hypometabolism involving the posterior part of the left orbito-frontal cortex, relative involvement of the adjacent cortex (gyrus rectus, anterior part of the left inferior frontal gyrus) and the temporal pole. This hypometabolism was retrospectively found on the previous PET but remained unconclusive. Note that the coregistration was imperfect on this examination, whereas it was almost perfect on the PET/MR, allowing a better 
confidence for the visual analysis. The patient was operated on without invasive monitoring despite a negative MRI. Surgery was based on a PET/MR guided cortical resection, including the hypometabolic orbito-frontal cortex and the adjacent inferior frontal gyrus, in front of Broca area. FCD type 2A was found in the cortical specimen. The patient has been seizure-free for 2 years and the antiepileptic drugs greatly reduced.
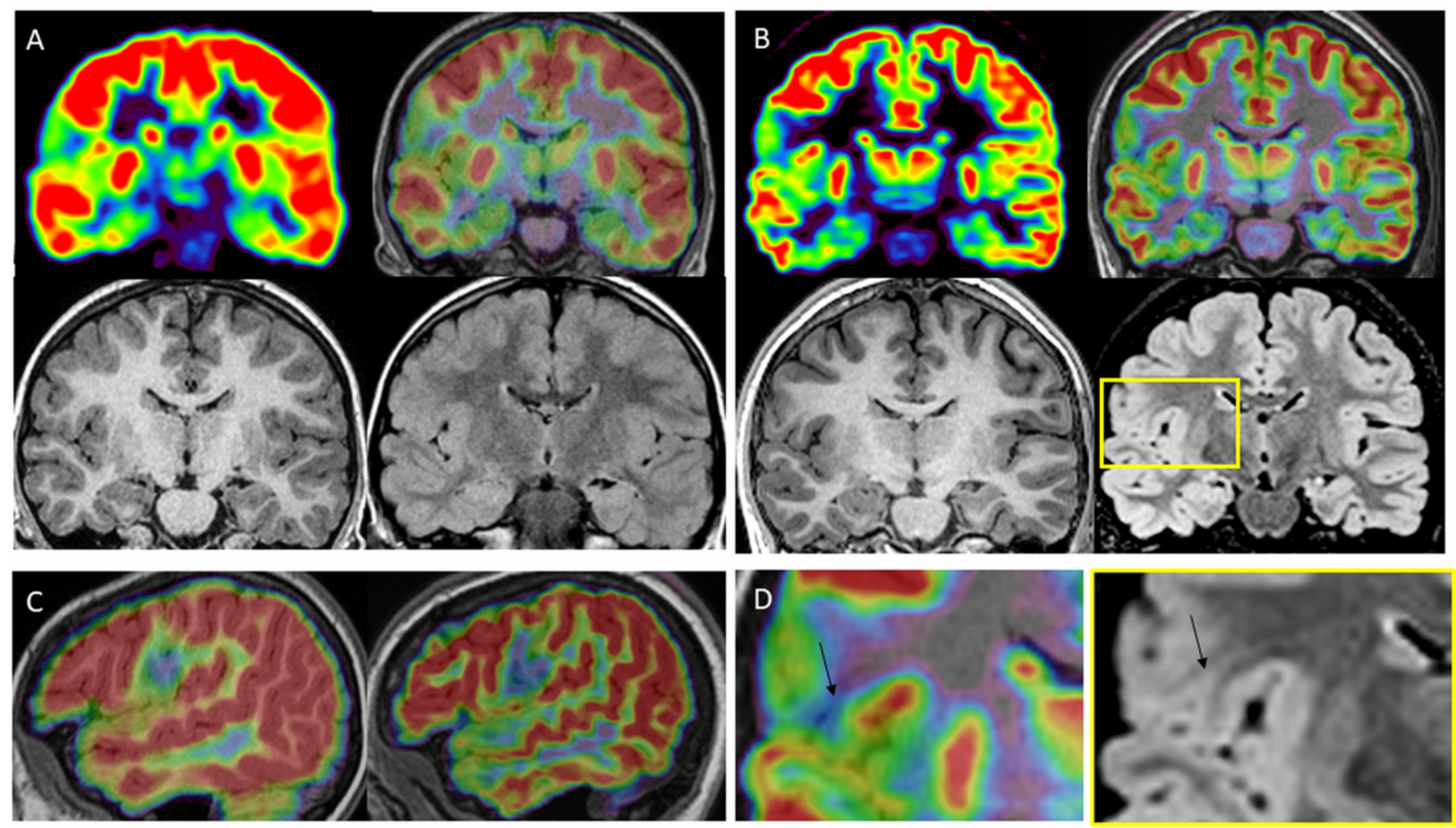

\section{Figure 2}

Comparison between PET+MR (A) and PET/MR (B); coronal plane, PET alone and co-registered on MRI; MRI: T1 and FLAIR sequences; sagittal view comparing the PET co-registered on MRI from the PET+MR and the PET/MR (C); magnification of PET and MR images from PET/MR (D). 13 year-old female, drug-resistant right fronto-opercular epilepsy since the age of 2 years; nocturnal seizures, 1-4/night. Right centro-temporal ictal onset, negative MRI. First PET at the age of 7 years, showing a focal hypometabolism involving the right opercular cortex, the inferior rolandic cortex and the insula. No clear-cut lesion was found on the corresponding area on MRI. Surgery was not indicated because of the wide epileptogenic zone presumed and the functional risks. The TEP/MR was performed 6 years later, showing a focal hypometabolism in the same area, however allowing to distinguish a severe hypometabolism well localised on the precentral operculum, associated with a less severe involvement of the adjacent cortex, sparing the rolandic cortex and the insula. Careful analysis of MRI, guided by the maximal site of hypometabolism on PET, allowed to identify a small gyrus with a thin extension toward the ventricle (transmantle sign, arrow), typical for FCD. The patient was operated on without invasive monitoring. The cortical resection was limited to the lesional cortex detected by the $\mathrm{PET} / \mathrm{MR}, \mathrm{FCD} 2 \mathrm{~A}$ was found in the cortical specimen. No neurological deficit occurred post-operatively. She has been seizure-free for 7 months (and antiepileptic drugs slightly reduced). 